\title{
Improving Lifetime of Multicore Soft Real-Time Systems through Global Utilization Control
}

\author{
Yue $\mathrm{Ma}^{1}$, Thidapat Chantem ${ }^{2}$, X. Sharon Hu${ }^{1}$, Robert P. Dick \\ ${ }^{1}$ Department of CSE, University of Notre Dame, Notre Dame, IN 46656 \\ ${ }^{2}$ Department of ECE, Utah State University, Logan, UT 84322 \\ ${ }^{3}$ Department of EECS, University of Michigan, Ann Arbor, MI 48109 \\ Email: \{yma1, shu\}@nd.edu, tam.chantem@usu.edu, dickrp@umich.edu
}

\begin{abstract}
System lifetime reliability is an important design consideration for many real-time embedded systems. Increasing integrated circuit power density and the subsequent rise in chip temperature negatively impact the lifetime reliability of such systems. Although existing thermal-aware methods are effective in reducing temperature, they may not increase, and may even hamper, the system lifetime reliability. The complicated relationship between temperature and system lifetime requires that reliability be considered explicitly during system design. This paper presents a reliability-aware utilization control framework for homogeneous multicore soft real-time systems. The framework employs a model predictive controller to increase the system lifetime by manipulating the processor utilization. An online heuristic algorithm is introduced to adjust the controller's sampling window in order to reduce the effects of thermal cycling on reliability. Simulation results show that the proposed approach can improve the system mean time to failure by at least $43 \%$ and as much as $369 \%$ compared to existing techniques.
\end{abstract}

\section{Categories and Subject Descriptors}

B.8.2 [PERFORMANCE AND RELIABILITY]: Performance Analysis and Design Aids

\section{Keywords}

System-level design; Reliability optimization; Dynamic voltage and frequency scaling

\section{INTRODUCTION}

Multicore systems provide high performance and power efficiency. However, due to CMOS technology scaling, multicore chips increasingly have higher power density and temperature, which, in turn, reduces system lifetime [5].

There have been a number of research efforts on increasing system lifetime by controlling temperature (e.g., $[2,10,12])$. However, since device lifetime is dependent not only on temperature, but also on temperature variation (thermal cy-

This work was supported in part by NSF under awards CNS-1319904, CNS-1319718, and CNS-1319784 and by Sandia National Laboratory.

Permission to make digital or hard copies of all or part of this work for personal or classroom use is granted without fee provided that copies are not made or distributed for profit or commercial advantage and that copies bear this notice and the full citation on the first page. Copyrights for components of this work owned by others than ACM must be honored. Abstracting with credit is permitted. To copy otherwise, or republish, to post on servers or to redistribute to lists, requires prior specific permission and/or a fee. Request permissions from permissions@acm.org. GLSVLSI'15, May 20-22, 2015, Pittsburgh, PA, USA.

Copyright (C) 2015 ACM 978-1-4503-3474-7/15/05 ...\$15.00.

http://dx.doi.org/10.1145/2742060.2742113. cling) [5], temperature reduction strategies alone are suboptimal for maximizing system mean time to failure (MTTF) [6]. Hence, a number of papers have proposed techniques to increase system MTTF directly $[1,3,4]$. Coskun et al. presented a reliability-aware job scheduling and power management approach for multicore systems [1]. However, their work does not consider real-time requirements. Based on wear sensors, Hartman et al. designed a run-time based task mapping algorithm to improve the MTTF of real-time systems [3]. Unfortunately, wear sensors are not yet widely available and can only detect a limited set of integrated circuit (IC) failure mechanisms [11]. Compared to these studies, our approach considers IC-dominant failure mechanisms to improve the lifetime reliability of soft real-time systems.

In this paper, we aim to improve the lifetime reliability of homogeneous multicore systems without sacrificing realtime performance. Since system MTTF depends on both temperature and thermal cycling, we introduce a reliabilityaware utilization control framework, called RUC, that jointly optimizes these two factors at the same time. RUC is composed of a global utilization control (GUC) to reduce temperature and a sampling window control (SWC) to reduce thermal cycling. Our main contributions are as follow.

(i) We show that increasing utilization via decreasing execution speed reduces runtime temperature. We exploit this observation to design a model predictive controller (MPC) that keeps the system utilization at a desired value (known as utilization set point) to improve the MTTF without increasing deadline miss rates. The design of MPC considers the real-time constraints as well as MTTF dependency on core temperatures.

(ii) An important parameter in MPC design is the sampling window length $\left(L_{s w}\right)$ which impacts system reliability as it affects both core temperatures and thermal cycles. A larger sampling window reduces thermal cycling but increases core temperatures. To achieve the desired trade off between temperature and thermal cycling, we introduce a heuristic to dynamically adjust $L_{s w}$ of the MPC.

We conducted a large set of simulations to assess the effectiveness of our approach. Compared to existing temperatureaware and utilization control mechanisms, our proposed framework achieves, on average, $43 \%$ improvement in MTTF, and up to $369 \%$ improvement, while allowing more task sets to satisfy real-time constraints.

\section{PRELIMINARIES}

We consider four main IC-dominant failure mechanisms in this paper: electromigration (EM), stress migration (SM), time dependent dielectric breakdown (TDDB), and thermal cycling (TC) [6]. Figs. 1(a), (b), and (c) depict the MTTF of an example system as a function of amplitude (e.g., the 


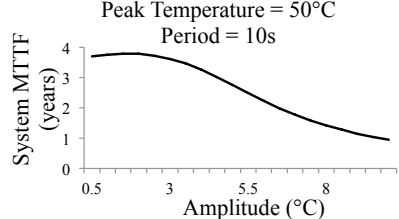

(a)

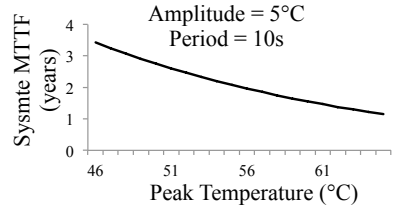

(c)

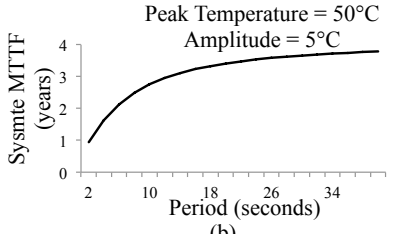

(b)

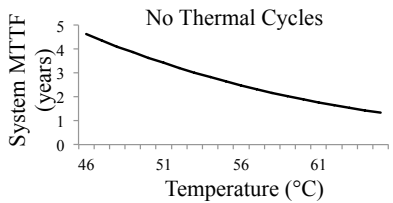

(d)
Figure 1: System MTTF due to: (a) amplitude of thermal cycle; (b) period of thermal cycles; (c) peak temperature of thermal cycles; and (d) temperature but no thermal cycles.

difference between the highest (peak) and lowest (valley) temperature), period, and peak temperature, of thermal cycles, respectively, according to a reliability modeling tool [13] with default settings. For comparison purposes, Fig.1 (d) shows the system MTTF due to temperature alone without thermal cycles. As can be seen from Fig.1, the system MTTF is generally higher with lower temperature and smaller thermal cycles, but the precise reliability model is somewhat more complicated.

We focus on homogeneous multicore systems in this paper. Let $M=\left\{\rho_{1}, \rho_{2}, \ldots, \rho_{m}\right\}$ denote the $m$ cores. We assume that these cores have identical thermal and electrical characteristics and initial wear state. The system MTTF of the multicore system is $\min \left\{M T T F\right.$ of core $\left.\rho_{i}\right\}, i=1, \ldots, m$, and the MTTF of core $\rho_{i}\left(M T T F_{i}\right)$ can be calculated based on the core's failure rate, or using existing reliability modeling tools, e.g., [13]. For the multicore system under consideration, it has been proven that the system MTTF is maximized if the power is distributed evenly among the cores [11].

Power consumption is the fundamental cause of rising temperature and hence system failure. When a core performs operations, it dissipates dynamic $\left(P_{d y n}\right)$ and leakage power $\left(P_{\text {leak }}\right)$, but when idle only consumes leakage power [10]. Suppose the utilization of a core in a given time interval $\Delta t$ is $U=\frac{\Delta t_{a}}{\Delta t}$, where $\Delta t_{a}$ is the amount of time that the core executes operations, the average power $(\bar{P})$ can be calculated as

$$
\begin{aligned}
\bar{P} & =U \times P_{d y n}+P_{\text {leak }} \\
& =U \times V^{\alpha_{0}} \times f \times \alpha_{1}+\left(\alpha_{2}(f, V)+\alpha_{3}(f, V) \times T\right) \times V,
\end{aligned}
$$

where $V$ and $f$ are the core's voltage and frequency, respectively. $\alpha_{0} \geq 1, \alpha_{1}>0$, and $\alpha_{2}, \alpha_{3}$ are some voltage/frequency dependent parameters [10].

In term of workloads, we assume that tasks are periodic and already mapped to cores and that no migration is allowed. Workloads may change over time. Tasks on each core are scheduled by a real-time scheduling policy such as earliest deadline first (EDF) or rate monotone (RM) [8]. In a soft real-time system, the late completion of tasks is acceptable but should be avoided. The job of the $j^{\text {th }}$ task is denoted by $\tau_{j}$, which is associated with a tuple $\left\{e_{j}, d_{j}\right\}$ where $e_{j}$ is the execution time and $d_{j}$ is the relative deadline (as well as the period). For a given time duration, thereafter referred to as sampling window $k\left(S W_{k}\right)$, if core $\rho_{i}$ 's frequency is fixed at $f_{i}(k)$, its utilization can be calculated as

$$
U_{i}(k)=\sum_{\tau_{j} \in \Gamma(k)} \frac{e_{j}}{d_{j}}=\sum_{\tau_{j} \in \Gamma(k)} \frac{e_{j}^{*}+e_{j}^{\prime} / f(k)}{d_{j}} .
$$

where $e_{j}^{\prime}$ reflects the portion of job execution that is dependent on the core's frequency, and $e_{j}^{*}$ is the independent portion. $\Gamma(k)$ is the set of jobs executed in $S W_{k}$.

\section{PROBLEM FORMULATION}

We will use a control theoretic approach to improve the system MTTF by controlling task utilization. Compared to temperature control and MTTF control, utilization control is easier to implement and already widely used in soft realtime systems $[9,12]$.

Theorem 1. If in a given time duration, $S W_{k}$, core temperatures are constant, then a higher frequency/voltage pair used in $S W_{k}$ leads to a higher temperature but a lower utilization.

Theorem 1 indicates that any method to control utilization through manipulations of core's frequency and utilization is also effective in temperature management. Before formulating the utilization control problem, we introduce some notation: $\bar{f}(k)$ is the average frequency over all cores; $U^{s}$ is the utilization set point; $U^{\max }$ is the upper bound on the utilization to ensure schedulability; $f_{\min }$ and $f_{\max }$ are the lowest and highest core's frequencies allowed, respectively. We aim to solve the following problem:

$$
\min \sum_{\rho_{i} \in M}\left(U^{s}-U_{i}(k)\right)^{2}
$$

The solution to (3) must satisfy the following constraints:

$$
\begin{cases}U_{i}(k) \leq U^{\max } & \text { for } \rho_{i} \in M \\ -f_{t h} \leq f_{i}(k)-\bar{f}(k) \leq f_{t h} & \text { for } \rho_{i} \in M, \text { and } \\ f_{\min } \leq f_{i}(k) \leq f_{\max } & \text { for } \rho_{i} \in M\end{cases}
$$

The first constraint ensures no cores exceed the schedulability bound. According to the system reliability discussion given in Section 2 and [11], the second constraint is introduced to bound the differences in the cores' frequencies, which in turn bound the temperature differences among the cores. The bound on frequency difference, $f_{t h}$, is typically set to $0.1 \mathrm{GHz}$. The third constraint is used to satisfy the cores' operating requirement.

\section{DESIGN OF RUC}

\subsection{RUC framework}

We propose a mechanism to improve system MTTF by solving the optimization problem in Equations (3)-(6) and dynamically adjusting the length of sampling window. We refer to the overall framework as RUC. RUC consists of two main components: global utilization control (GUC) and sampling window control (SWC). GUC reduces temperature by using an MPC, which dynamically adjusts core frequencies to enforce the utilization set point. SWC minimizes thermal cycles by dynamically adjusting $L_{s w}$.

At the end of $S W_{k}$, the utilization of each core is measured. Based on the utilization and MPC settings, MPC solves the optimization problem defined in Equations (3)(6). The solution consists of the cores' frequencies in the next sampling window and is sent to each core before the start of $S W_{k+1}$. At the end of $S W_{k}$, the measured temperature values are sent to and saved in SWC. Specifically, the temperature measured in the most recent $s$ sampling windows are saved. We refer to the $s$ sampling windows as one profiling window. At the end of each profiling window, a temperature profile is generated. SWC then analyzes this temperature profile and determines whether to increase or reduce the sampling window length in the next profiling window. 


\subsection{Global utilization control}

In this section, we discuss our GUC design to solve the constrained optimization problem in Equations (3)-(6) using an MPC. The basic idea behind any MPC is to optimize a cost function. Hence, we first make a comparison between the constrained optimization problem in Equations (3)-(6) and an MPC cost function optimization problem. After this, we transform the MPC cost function optimization problem to a standard quadratic programming problem to be solved by some existing solvers.

According to control theory, the design of MPC is to minimize a cost function for a given $S W_{k}$,

$$
J(k)=\sum_{i=N_{1}}^{N_{2}} \delta_{i}[U(k+i)-\rho(k+i)]^{2}+\sum_{j=1}^{N_{u}} \lambda_{j}\left[\frac{1}{\Delta f(k+j-1)}\right]^{2},
$$

where $N_{1}, N_{2}, N_{u}, i$, and $j$ are integers, $\delta_{i}$ is the tracking error weight, and $\lambda_{i}$ is the control penalty error [12]. The user specified reference trajectory $\rho(k+i)$ defines the ideal trajectory along which the utilization should converge to the set point. The first term in the cost function (7) is a variation of the function in Equation (3). The second term in Equation (7) minimizes the changes in the manipulated variable and does not affect the final result of the optimization problem. Hence, minimizing the cost function (7) under the constraints in Equations (4)-(6) would also lead to the optimal solution to the problem defined in Equations (3)-(6).

We propose using a quadratic programming solver to solve the optimization problem. A standard quadratic programming problem can be written as

$$
\min _{\varepsilon}\left\{\frac{1}{2} \varepsilon^{T} \Omega \varepsilon+\zeta^{T} \varepsilon\right\}, \text { s.t. }\left\{\begin{array}{l}
A \times \varepsilon \leq b \\
A e q \times \varepsilon=b e q \\
l b \leq \varepsilon \leq u b
\end{array}\right.
$$

where $\Omega, A$, and $A e q$ are matrices, $\zeta, b, b e q, l b$, and $u b$ are vectors, and $\varepsilon$ denotes the change in a core's frequency. The key point in MPC design is to transform the problem defined in Equations (4)-(7) to the standard quadratic programming format in Equation (8). Based on $\varepsilon$ and the core's frequency inside $S W_{k}$, the core's frequency at $S W_{k+1}$ can be directly calculated. We omit the details of the transformation to save space. After the transformation, we can directly solve the quadratic programming problem using a standard solver, e.g., quadprog tool in Matlab.

\subsection{Sampling window control}

The proposed GUC aims to reduce the temperature of the entire multicore chip and to balance the temperature differences among cores, but may introduce thermal cycles. Since a core's frequency and voltage can change from one sampling window to another, sampling window length, $L_{s w}$ directly impacts thermal cycles. It is difficult to precisely model how the sampling window length affects system reliability, and the complicated reliability model makes finding the best sampling window too time consuming for online use. Hence, to judiciously balance the impact of temperature against that of thermal cycles on system MTTF, we design an efficient online heuristic based on binary search to adjust $L_{s w}$ at runtime.

Since a core's temperature is constant inside each sampling window if a core's frequency is fixed, the aging effect of thermal cycles can be ignored if we only consider a single sampling window. In SWC, we use the concept of profiling window to adjust $L_{s w}$. A profiling window is composed of $s$ sampling windows with the same length and whose $s$ temperature points, each corresponding to the temperature of the respective sampling window, make up a temperature profile. At the end of each profiling window, we estimate the system MTTF from the temperature profile using a reliability modelling tool [13]. In our experimental evaluations,

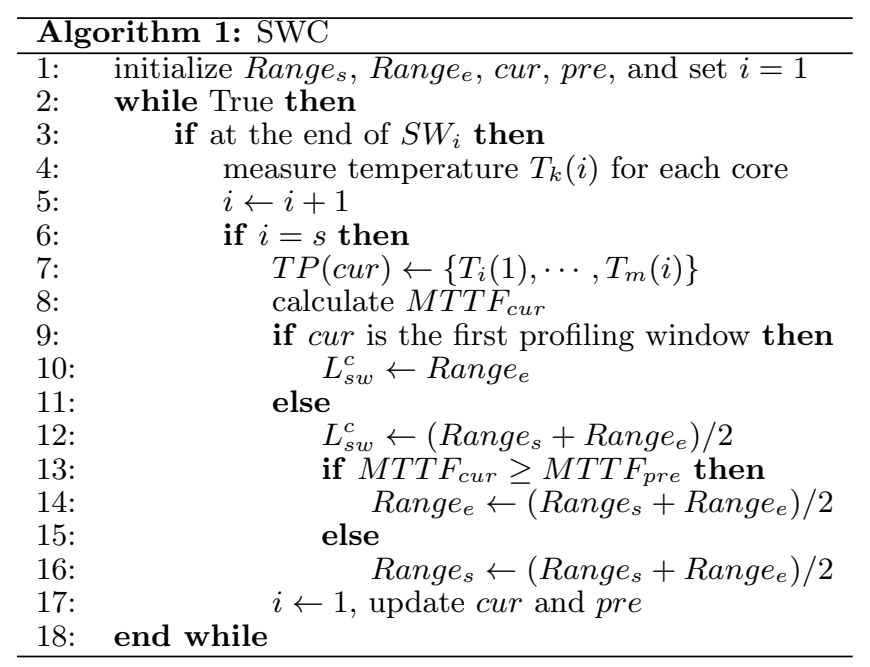

we find that $s=100$ achieves an precise system MTTF with an acceptable overhead in MTTF calculation. We store and compare the system MTTF in current and previous sampling window, and use the binary search to find the most appropriate sampling windows length.

To find $L_{s w}$, let $\left[\right.$ Range $_{s}$, Range $\left._{e}\right]$ denote the binary search range. We initially set Range $_{s}$, which is also the lower bound of $L_{s w}$, to 1 second to keep the overhead due to RUC under $1 \%$ of the sampling window length (based on an Intel Core i5 $2.6 \mathrm{GHz}$ and $2 \mathrm{~GB}$ memory). To determine an initial value for Range $_{e}$, we observe that since GUC expects a constant temperature in a given sampling window, so the sampling window length must be smaller than some constant $C_{H W}$, which depends on runtime environment and hardware platform and is typically on the order of 10 seconds. Moreover, since different hyperperiods $(H P \mathrm{~s})$ of a given periodic task set have the same workload, a core's frequency remains the same across different $H P \mathrm{~s}$ if $L_{s w}=H P$ and utilization set point is fixed. Since a larger $L_{s w}$ leads to higher temperature due to slow frequency scaling, the appropriate value for $L_{s w}$ should be less than or equal to $H P$. As a result, we initialize $R_{\text {ange }}$ to $\min \left\{H P, C_{H W}\right\}$. The pseudo code for SWC is given in Alg.1, where $L_{s w}^{c}$ is $L_{s w}$ in the current sampling window, and cur and pre represent the current and previous profiling window. The search ranger, $\left[\right.$ Range $_{s}$, Range $\left._{e}\right]$, is updated in each iteration until the most appropriate value for $L_{s w}$ is found.

\section{EVALUATION}

We have conducted a large number of simulation runs to evaluate the effectiveness of RUC. An event-driven simulator was implemented to simulate task execution. Tasks are scheduled using EDF [8] and executed to completion even if they have missed their deadlines. Each benchmark is composed of 50 randomly generated task sets. In each benchmark, the utilization level is kept constant. The utilization levels of the task set considered are $50,60, \ldots, 90 \%$. For the task set $\Gamma$, the utilization level is defined to be $U_{\text {level }}=\sum_{\tau_{i} \in \Gamma} \frac{e_{i}}{d_{i}}$. It is not necessary to evaluate the effectiveness of RUC under low utilization levels since the lowest frequency level would allow all the deadlines to be met while minimizing the core temperatures. To model different task behaviors, we studied two cases: fixed and variable task execution times. For the latter case, actual execution times of jobs are obtained by multiplying the specified execution times by a random value in the range $[0.9,1.1]$.

The hardware platform consists of 4 homogeneous cores and each of them is an ALPHA 21264 microprocessor, since 


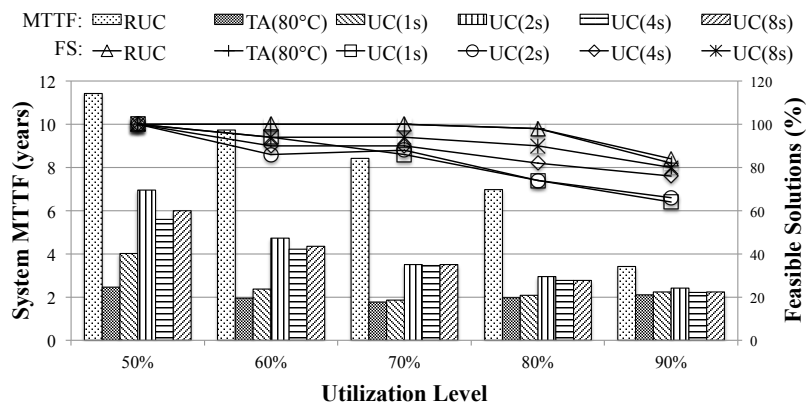

Figure 2: MTTF and FS values obtained by RUC, UC, and TA when tasks' execution times are fixed.

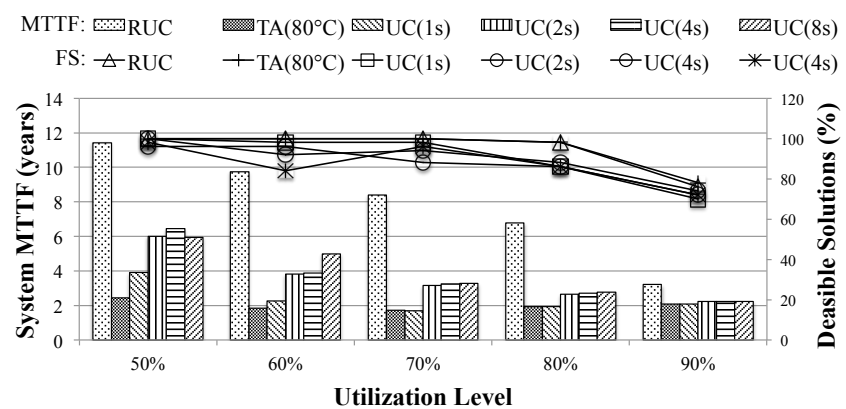

Figure 3: MTTF and FS values obtained by RUC, UC, and TA when tasks' execution times vary dynamically.

it is the only publicly available processor with well established power and thermal models [10]. The processor parameters and constants are listed in Table I. The initial temperature is assumed to be $330^{\circ} \mathrm{F}\left(56.85^{\circ} \mathrm{C}\right)$ and the ambient temperature is $318.15^{\circ} \mathrm{F}\left(45^{\circ} \mathrm{C}\right)$. The runtime temperature is calculated using Hotspot [7].

Table 1: Processor Parameters and Constants [10]

\begin{tabular}{|c|c|c|c|c|}
\hline Voltage (V) & Frequency (GHz) & $\alpha_{1}$ & $\alpha_{2}$ & $\alpha_{3}$ \\
\hline 0.7 & 1.00 & 1.422 & 0.1903 & 15.0 \\
\hline 0.8 & 1.25 & 2.916 & 0.2111 & 15.0 \\
\hline 0.9 & 1.5 & 5.41 & 0.234 & 15.0 \\
\hline 1.0 & 1.75 & 9.702 & 0.2592 & 15.0 \\
\hline 1.1 & 2.00 & 17.22 & 0.2867 & 15.0 \\
\hline
\end{tabular}

We compare the performance of RUC against two representative controller based mechanisms: utilization control (UC) and temperature-aware (TA). Similar to the existing work [9], UC controls a core's frequency to force the utilization to converge to a chosen set point, but it has a fixed $L_{s w}$. In our simulations, $L_{s w}$ of 1 second (UC(1s)), 2 seconds (UC(2s)), 4 seconds (UC(4s)) and 8 seconds (UC( $8 \mathrm{~s})$ ) are used. As for TA [12], each core's temperature is controlled to converge to its chosen set point. The temperature set point is $80^{\circ} \mathrm{C}$, which is below the temperature threshold for hardware throttling [12]. We compare RUC and TA to show that temperature reduction alone is suboptimal in maximizing system MTTF.

We compare RUC, UC and TA in terms of MTTF and real-time performance. Real-time performance is measured by the percentage of feasible solutions (FS), which is the ratio of the number of task sets satisfying their real-time requirements over the total number of task sets. For UC and RUC, the utilization set point is 90\% [9]. When tasks execution times are fixed (see Fig.2), the average MTTF values due to RUC for the 5 benchmarks are 11.42, 9.73, 8.43, 6.98, and 3.42 years, respectively. RUC increases MTTF values by up to nearly $364 \%$ and at least $52 \%$. In addition, using RUC always results in the highest FS compared to the other mechanisms in all the benchmarks. These results show that by controlling utilization and adjusting the sampling window length, more real-time task sets can be feasibly scheduled while maximizing system lifetime. RUC exhibits a similar improvement when tasks' execution times vary at runtime (see Fig.3). Its average MTTF values are 11.43, 9.74, 8.40, 6.78 , and 3.22 years for the five benchmarks. RUC improves system MTTF by $43 \%-369 \%$ while maintaining FS at a high level. We can conclude that for a variety of different runtime environments, RUC improves system lifetime and increases the schedulability of real-time tasks.

\section{CONCLUSIONS}

We proposed a reliability-aware utilization control framework to maximize the lifetime of multicore systems under soft real-time constraints. After observing the relationship between reliability and temperature, our mechanism jointly minimizes core temperatures, the temperature differences among cores, and thermal cycling. It uses a model predictive controller to control the core utilization by adjusting a core's frequency/voltage within each sampling window. We also introduced a heuristic to dynamically determine the length of a sampling window. Simulation results reveal that our approach is indeed effective in increasing the lifetime of soft real-time systems. As future work, we plan to extend our mechanism to heterogeneous multicore and hard real-time systems.

\section{References}

[1] A. Coskun, et al. Evaluating the impact of job scheduling and power management on processor lifetime for chip multiprocessors. In Proc. Int. Conf. Measurement and Modeling of Computer System, pages 169-180, Jun. 2009.

[2] T. Chantem, R. P. Dick, and X. Hu. Temperature-aware scheduling and assignment for hard real-time applications on MPSoCs. IEEE Trans. VLSI Systems, 19(10):1884 -1897 , Oct. 2011.

[3] A. Hartman and D. Thomas. Lifetime improvement through runtime wear-based task mapping. In Proc. Int. Conf. Hardware/Software Codesign and System Synthesis, pages 13-22, Oct. 2012.

[4] A. Hartman, D. Thomas, and B. Meyer. A case for lifetime-aware task mapping in embedded chip multiprocessors. In Proc. Int. Conf. Hardware/Software Codesign and System Synthesis, pages 145-154, Oct. 2010

[5] J. Srinivasan, et al. The impact of technology scaling on lifetime reliability. In Proc. Int. Conf. Dependable Systems and Networks, pages 177-186, Jun. 2004.

[6] JEDEC Solid State Technology Association. Failure mechanisms and models for semiconductor devices. JEDEC Publication, 2003.

[7] K. Skadron, et al. Temperature-aware microarchitecture: Modeling and implementation. ACM Trans. Architecture and Code Optimization, 1(1):94-125, Mar. 2004.

[8] C. Liu and J. Layland. Scheduling algorithm for multiprogramming in a hard-real-time environment. J. of ACM, 20(1):46-61, Jan. 1973.

[9] C. Lu, X. Wang, and X. Koutsoukos. Feedback utilization control in distributed real-time systems with end-to-end tasks. IEEE Trans. Parallel and Distributed Systems, 16(6):550-561, Jun. 2005.

[10] G. Quan and V. Chaturvedi. Feasibility analysis for temperature-constraint hard real-time periodic tasks. IEEE Trans. Industrial Informatics, 6(3):329-339, Aug. 2012.

[11] T. Chantem, et al. Enhancing multicore reliability through wear compensation in online assignment and scheduling. In Proc. Design, Automation and Test in Europe, pages 1373-1378, Mar. 2013.

[12] Y. Fu, et al. Feedback thermal control of real-time systems on multicore processors. In Proc. Int. Conf. Embedded Software, pages 113-122, Oct. 2012.

[13] Y. Xiang, et al. System-level reliability modeling for MPSoCs. In Proc. Int. Conf. Hardware/Software Codesign and System Synthesis, pages 297-306, Oct. 2010. 